\title{
Artigo/Article
}

\section{Suscetibilidade de larvas de Culex quinquefasciatus a diferentes inseticidas}

\author{
Susceptibility of Culex quinquefasciatus larvae to different insecticides
}

Stênio Nunes Alves ${ }^{1}$, Jacqueline Domingues Tibúrcio ${ }^{1}$ e Alan Lane de Melo²

\section{RESUMO}

Introdução: O presente estudo teve como objetivo avaliar a suscetibilidade de larvas de Culex quinquefasciatus a dois piretróides (Cipermetrina e Deltametrina), dois derivados da Avermectina (ivermectina e abamectina) e a um organofosforado (Temefós). Métodos: Larvas de $3^{\circ}$ e $4^{\circ}$ instares de $C$. quinquefasciatus foram expostas a diferentes concentrações destes (onze repetições) seguindo o protocolo da Organização Mundial de Saúde. Uma hora após a exposição, as larvas foram lavadas em água desclorada, transferidas para recipientes plásticos contendo água sem cloro, alimentadas e observadas por períodos de $24 \mathrm{~h}$, até se transformarem em adultos. Para a determinação das concentrações letais, os valores foram submetidos à análise de regressão usando o modelo probit pelo programa Minitab 15. Resultados: Diferenças entre as estimativas da $\mathrm{CL}_{50}$ e $\mathrm{CL}_{90}$ justificaram que a população de mosquitos testada apresenta heterogeneidade em resposta aos inseticidas, sendo a maior concentração utilizada para a $\mathrm{CL}_{50}$, a partir da análise de probit para o Temefós. Todos os inseticidas avaliados causaram mortalidade mais acentuada nas primeiras $24 \mathrm{~h}$ exceto quando expostas à ivermectina. Conclusões: As larvas são suscetíveis a todos os inseticidas testados e há uma necessidade de um monitoramento dos inseticidas utilizados.

Palavras-chaves: Culex. Inseticidas. Temefós. Piretróides. Avermectinas.

\begin{abstract}
Introduction: This study aimed to assess the susceptibility of Culex quinquefasciatus larvae to two pyrethroids (Cypermethrin and Deltamethrin), two derivatives of Avermectin (Ivermectin and Abamectin) and an organophosphate (Temephos). Methods: Third- and fourth-instar larvae of $C$. quinquefasciatus were exposed to different concentrations of insecticides (eleven repetitions) according to the World Health Organization's protocol. One hour after exposure, larvae were washed in dechlorinated tap water, transferred to plastic containers containing water without chlorine, fed and observed for periods of $24 \mathrm{~h}$ until reaching adulthood. To determine lethal concentrations, the obtained values were submitted to regression analysis using the probit model with the Minitab 15 program. Results: The highest concentration used for the $\mathrm{LC}_{50}$ from probit analysis was for Temephos. The evaluated insecticides caused more pronounced larvae mortality in the first $24 \mathrm{~h}$ with the exception of those exposed to ivermectin. Conclusions: This study demonstrates that larvae are susceptible to all tested insecticides and that there is a need for monitoring the use of insecticides.
\end{abstract}

Keywords: Culex. Insecticides. Temephos. Pyrethroids. Avermectins.

\footnotetext{
1. Campus Centro-Oeste Dona Lindu, Universidade Federal de São João del-Rei, Divinópolis, MG. 2. Departamento de Parasitologia, Instituto de Ciências Biológicas, Universidade Federal de Minas Gerais, Belo Horizonte, MG.

Endereço para correspondência: Dr. Stênio Nunes Alves. Rua Sebastião Gonçalves Coelho 400, Chanadour, 35501-296 Divinópolis, MG.

Tel: 5537 3221-1831

e-mail: stenioalves@ufsj.edu.br

Recebido para publicação em 02/09/2010

Aceito em 19/04/2011
}

\section{INTRODUÇÃO}

De hábitos antropofílicos e endofílicos, particularmente em regiões urbanas, Culex quinquefasciatus Say, 1823 apresenta ampla distribuição geográfica. Suas larvas são capazes de se desenvolverem em diversos tipos de habitats, de esgotos à água limpa, com preferência para os primeiros ${ }^{1-3}$. Por estar relacionado à transmissão da filariose bancroftiana nas Américas, e em particular no Brasil, este inseto apresenta grande importância em saúde pública, gerando desafios administrativos e públicos para o seu controle ${ }^{4}$.

Inseticidas organoclorados, organofosforados, carbamatose piretróidestêm sidolargamente empregados para controlar mosquitos vetores de doenças em diversas partes do mundo. Entretanto, em alguns lugares, organofosforados e carbamatos têm sido ineficazes para o controle efetivo contra C. quinquefasciatus ${ }^{5}$. $\mathrm{Na}$ Ásia, vários graus de tolerância/resistência de caráter tóxico de fenitrotion e vários derivados de carbamatos e piretróides têm sido documentados para esta espécie ${ }^{6}$. Na América Latina, existem relatos de resistência a organofosforados e carbamatos em populações adultas de C. quinquefasciatus ${ }^{4,7-9}$.

A resistência a inseticidasé um problema crescente nos programas de controles de vetores. Por outro lado, o contínuo monitoramento das populações de mosquito pode ter um importante papel na tentativa de avançar com estratégias de manejo que prevenirão ou minimizarão o desenvolvimento de resistência a inseticidas efetivo ${ }^{10}$, assim como a utilização de novos produtos para o combate aos insetos.

O presente trabalho teve como objetivo avaliar a suscetibilidade de larvas de $C$. quinquefasciatus a dois piretróides (cipermetrina e deltametrina), dois derivados da ivermectina (ivermectina e abamectina) e um organofosforado (Temefós).

\section{MÉTODOS}

\section{Insetos}

As formas imaturas de C. quinquefasciatus foram obtidas da criação semi-natural mantida no Laboratório de Taxonomia e Biologia de Invertebrados do Departamento de Parasitologia 
no Instituto de Ciências Biológicas da Universidade Federal de Minas Gerais. Para tanto, as posturas obtidas foram transferidas para cubas plásticas ( $50 \times 40 \times 25 \mathrm{~cm})$ contendo 251 de água de torneira desclorada. Após a eclosão, as larvas foram alimentadas com ração para camundongos da marca Purina. A criação foi feita sob condições naturais de temperatura e fotoperíodo ${ }^{11}$.

\section{Inseticidas}

A partir de soluções estoques preparadas nos dias dos ensaios, foram utilizados o organofosforado Temefós (Fersol 500CE) nas concentrações de 125.000, 62.500, 625, 125 e 6,25ppb; os piretróides cipermetrina (Fersol 200CE) nas concentrações de 50.000, 5.000, $500,250,50,25,5,2,5$ e 0,5ppb, e deltametrina (Fersol 25CE) $6.250,625,62,5,31,25,6,25,3,125$ e 0,3125ppb, e as avermectinas ivermectina (Ivomec 1\%p/v, Merial), concentrações de 500, 100, 50,10 e 1,25ppb e abamectina (Vertimec 18CE, Syngenta) 18.000, $1.800,180,18$ e $1,8 \mathrm{ppb}$

As concentrações foram estabelecidas a partir daquelas que apresentaram resultados para análise de probit.

\section{Bioensaio}

Foram utilizadas 3.520 larvas de $3^{\circ}$ e $4^{\circ}$ instares de C. quinquefasciatus expostas às diferentes concentrações dos inseticidas supracitados durante $1 \mathrm{~h}$ para se conseguir a melhor concentração sub-letal. Em cada recipiente plástico que continha $100 \mathrm{ml}$ de solução foram acrescentadas 10 larvas. Os testes apresentaram onze repetições, em dias diferentes e seguiram o protocolo da Organização Mundial de Saúde ${ }^{12}$. Uma hora após a exposição, as larvas foram lavadas em água de torneira desclorada, transferidas para recipientes plásticos contendo água sem cloro, alimentadas com ração para camundongos e observadas por períodos de $24 \mathrm{~h}$, até se transformarem em adultos. Todo o experimento foi conduzido em temperatura de $26 \pm 1^{\circ} \mathrm{C}$ e fotoperíodo de $12 \mathrm{~h}$.

\section{Análise estatística}

Os valores para a determinação das concentrações letais foram submetidos à análise de regressão usando o modelo probit $^{13}$ pelo programa Minitab 15 . Em todos os modelos ajustados, o efeito das doses foi avaliado como significativo se valor-p<0,05. A qualidade do ajuste dos modelos foi verificada pela análise dos resíduos através de gráficos diagnósticos e pela estatística Deviance, utilizando o teste qui-quadrado. Diferenças significativas (valor- $\mathrm{p}<0,05)$ foram consideradas como falta de ajuste do modelo aos dados observados.

\section{RESULTADOS}

$\mathrm{O}$ modelo ajustado, as concentrações letais estimadas $\mathrm{CL}_{50} \mathrm{e}$ $\mathrm{CL}_{95}$, os respectivos intervalos de confiança à $95 \%$ e a qualidade do ajuste dos modelos estão demonstrados na Tabela 1. Pode-se verificar que todos os modelos foram significativos $(p<0,05)$ e, exceto para ivermectina, todos se mostraram bem ajustados ( $p>0,05)$. As diferenças entre as estimativas da $\mathrm{CL}_{50}$ e $\mathrm{CL}_{95}$ indicam que a população de mosquitos testada apresenta heterogeneidade em resposta aos inseticidas.

Os testes efetuados em larvas de C. quinquefasciatus procurando determinar as concentrações letais (CL) resultaram em diferentes concentrações para os inseticidas com índice de mortalidade para as menores concentrações que variaram de $2 \%$ para abamectina a $1,8 \mathrm{ppb}$ a $39 \%$ para ivermectina a $1,25 \mathrm{ppb}$ (Figura 1). No entanto, a maior concentração utilizada para a $\mathrm{CL}_{95}$, a partir da análise de probit, foi a utilizada para a deltametrina (Tabela $\mathbf{1}$ ).

Verificou-se que o Temefós, os piretróides e a abamectina causaram mortalidade mais acentuada nas primeiras $24 \mathrm{~h}$ ao contrário daquelas expostas à ivermectina (Figura 1).

TABELA 1 - Concentração letal (CL) para os inseticidas em ppb, para larvas de Culex quinquefasciatus após 1 h de exposição.

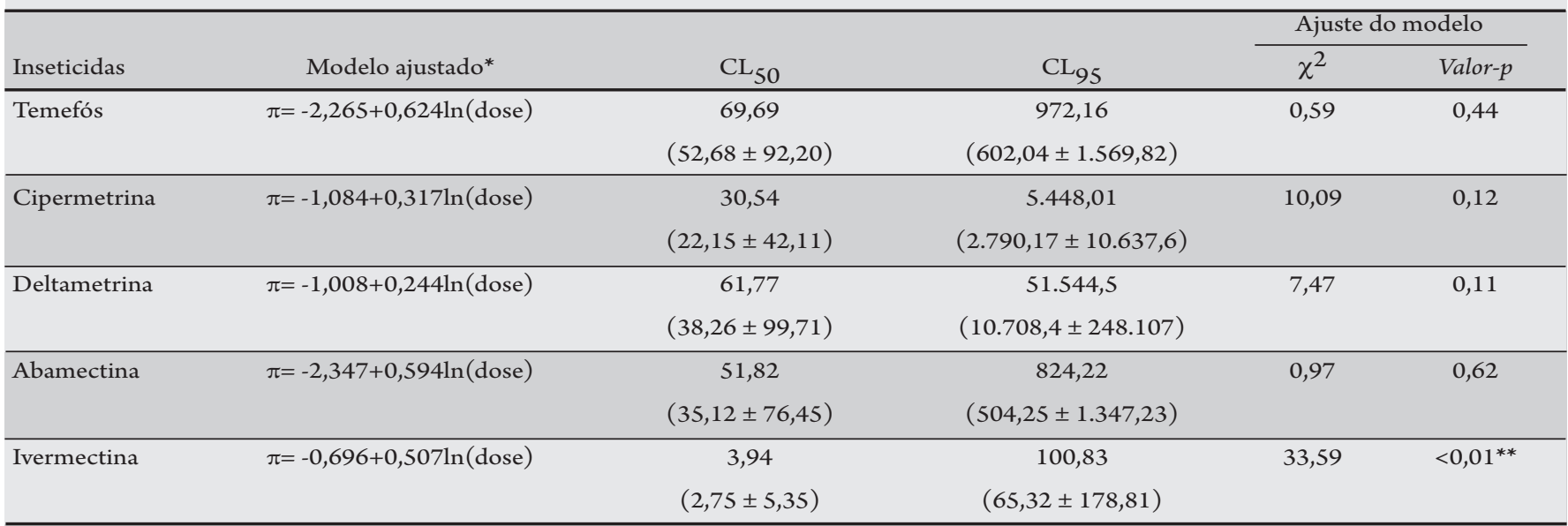

${ }^{*}$ Todos os coeficientes do modelo ajustado foram significativos $(\mathrm{p}<0,05),{ }^{* *}$ Estatística Deviance: valor-p $<0,05$ indica falta de ajuste do modelo aos dados observados. 


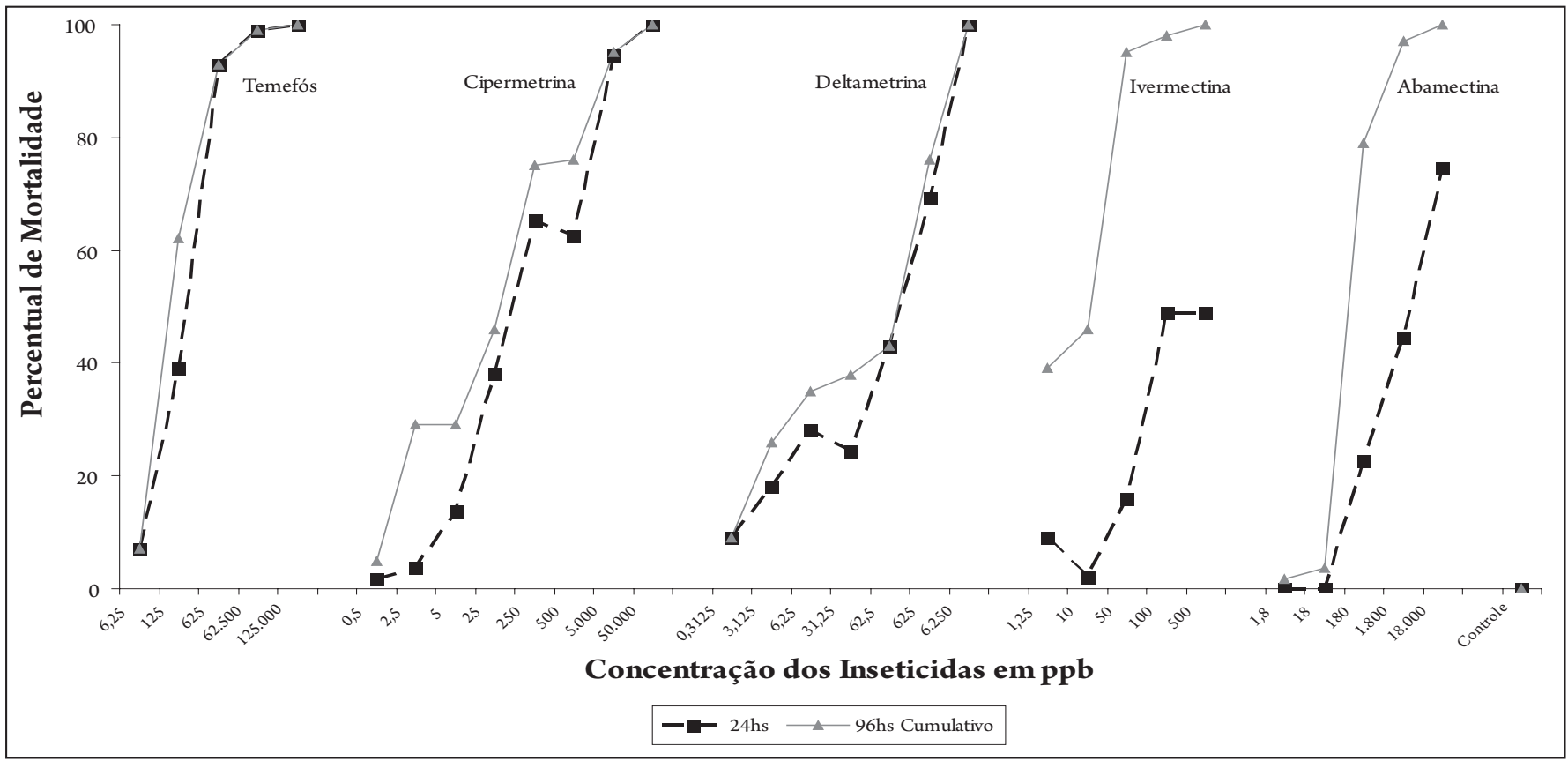

FIGURA 1 - Percentual de mortalidade por tempo de larvas de C. quinquefasciatus após 1 h de exposição a diferentes concentrações de Inseticidas em ppb.

\section{DIsCUsSÃO}

Os dados obtidos no presente estudo indicam necessidade de uma dosagem muito alta de deltametrina para produzir um alto índice de mortalidade de larvas sugerindo resistência destas ao inseticida utilizado. Tal resultado contrasta com estudo anterior que verificou

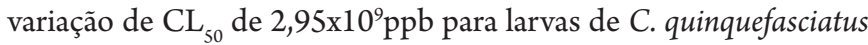
obtidas em laboratório a $7,77 \times 10^{7} \mathrm{ppb}$ para aquelas oriundas de campo, muito menor que a $\mathrm{CL}_{50}$ obtida no presente trabalho ${ }^{14}$.

Os resultados aqui obtidos indicam também que as larvas podem estar resistentes à cipermetrina e ao Temefós. Considerouse para isso que registros de letalidade com cipermetrina para essa espécie são maiores em concentrações menores: $\mathrm{CL}_{50}=0,0016 \mathrm{ppm}$ inseticida ativo (i.a.) para larvas de uma linhagem suscetível e $\mathrm{CL}_{50}=0,004 \mathrm{ppm}$ i.a. para uma linhagem parental com resistência em populações cubanas ${ }^{15}$ e registro de uma $\mathrm{CL}_{50}=0,003 \mathrm{ppm}$ e uma $\mathrm{CL}_{90}=0,01 \pm 0,006 \mathrm{ppm}$ i.a. deste piretróide para uma colônia resistente de C. quinquefasciatus, no Rio de Janeiro, quando comparada com a $\mathrm{CL}_{50}=0,0008 \mathrm{ppm}$ da linhagem referência ${ }^{16}$. Um fator que contribui para que a resistência possa ser evidenciada para Temefós é a $\mathrm{CL}_{50}$ utilizada no presente estudo ser 35 vezes maior que a preconizada pela $\mathrm{WHO}^{6}$ para este inseto.

A resistência de algumas linhagens de larvas de dípteros à inseticidas tem sido atribuída à ação de enzimas destoxificadoras ${ }^{16,17}$. Entre os mecanismos envolvidos na resistência aos piretróides, encontram-se o processo de destoxificação pela monoxigenase citocromo P450 ou por esterases ${ }^{18,19}$, que não confere resistência sistemática aos organoclorados, que são preferencialmente metabolizados pela glutationa-S-transferase ${ }^{20}$. A mortalidade menor verificada nas $24 \mathrm{~h}$ posteriores a exposição aos inseticidas sugere a presença de destoxificação nas larvas.

Por outro lado, a maior mortalidade entre 24 e $96 \mathrm{~h}$ após a exposição dos insetos aos derivados da Avermectina sugere uma ação residual maior destes produtos.
Embora no presente trabalho não tenha sido verificado o mecanismo metabólico envolvido na resistência aos inseticidas, modificações de esterases em populações brasileiras de C. quinquefasciatus para organofosforados já foram relatadas ${ }^{4,9,21}$. Entretanto, para os derivados da Avermectina, que geralmente são usados como antiparasitários em bovinos, equinos e ovinos e também como pesticidas agrícolas ${ }^{22-24}$, ainda não há registros na literatura de resistência no Brasil.

De fato, temos poucos relatos de utilização de derivados de avermectina em larvas de mosquitos no Brasil. Neste sentido, em estudos anteriores foram verificados em larvas de C. quinquefasciatus paralisia progressiva e altos níveis de mortalidade, provavelmente devido a ação bloqueadora da ivermectina nas junções neuromusculares ${ }^{25}$. Verificaram-se também alterações no corpo gorduroso das larvas sugerindo sinal de mobilização da reserva energética em resposta ao estresse larval quando estas foram expostas à solução de ivermectina ${ }^{26,27}$, altos níveis de mortalidade larval e diminuição da performance de adultos originados de geração larval, sendo possível neste caso estar ocorrendo um processo de destoxificação $0^{16,17}$.

Tais resultados sugerem que os piretróides devam ser utilizados com certo cuidado, já que o aumento dos registros de espécies de artrópodes resistentes aos piretróides, além da possibilidade de resistência cruzada com os organoclorados, é preocupação em todo o mundo, necessitando avaliações periódicas referentes ao seu emprego $^{28,29}$. Assim, monitoramento da efetividade dos inseticidas a serem usados em programas de controle contra vetores, bem como a utilização de outros pesticidas no combate aos mosquitos devem ser implementadas tendo em vista que mudanças da $\mathrm{CL}_{50}$ não acompanhadas por mudanças na $\mathrm{CL}_{95}$ podem ser manejadas com a troca temporária do produto ${ }^{30}$.

Pode-se concluir que as larvas são suscetíveis a todos os inseticidas testados quando em concentrações elevadas e que há uma necessidade de um monitoramento dos inseticidas utilizados. 


\section{CONFLITO DE INTERESSE}

Os autores declaram não haver nenhum tipo de conflito de interesse no desenvolvimento do estudo.

\section{SUPORTE FINANCEIRO}

Fundação de Amparo a Pesquisa do Estado de Minas Gerais. Este estudo foi financiado pelo Conselho Nacional de Pesquisa como parte da Tese de S. N. Alves.

\section{REFERÊNCIAS}

1. Consoli RAGB, Lourenço RO. Principais mosquitos de importância sanitária no Brasil. Rio de Janeiro: Fundação Instituto Oswaldo Cruz; 1994.

2. Forattini OP. Culicidologia Médica: Identificação, Biologia, Epidemiologia Volume II. São Paulo: Editora da Universidade de São Paulo; 2002.

3. Subra R. Biology and control of Culex pipiens quinquefaciatus Say, (Diptera, Culicidae) with special reference to Africa. Insect Sci Appl 1981; 1:319-338.

4. Campos J, Andrade CFS. Susceptibilidade larval de populações de Aedes aegypti e Culex quinquefasciatus a inseticidas químicos. Rev Saude Publica 2003; 37:523-527.

5. Sathantriphop S, Ketavan C, Prabaripai A, Visetson S, Bangs MJ, Akratanakul P, et al. Susceptibility and avoidance behavior by Culex quinquefasciatus Say to three classes of residual insecticides. J Vector Ecol 2006; 31:266-274.

6. World Health Organization. Vector resistance to pesticides. Fifteenth report of the expert committee on Vector biology and control. WHO Tech Rep Ser 1992; 818:1-55

7. Yébakima A, Yp-Tcha MM, Reiter P, Bisset J, Delay B, Chevillon C, et al. Detoxifying esterases in Culex pipiens quinquefasciatus from the Caribbean countries. J Am Mosq Control Assoc 1995; 11:363-366.

8. Bracco JE, Dalbon M, Marinotti O, Barata JMS. Resistência a inseticidas organofosforados e carbamatos em população de Culex quinquefasciatus. Rev Saude Publica 1997; 31:182-183.

9. González T, Bisset JA, Díaz C, Rodríguez MM, Brandolini MB. Insecticide resistance in a Culex quinquefasciatus strain from Rio de Janeiro, Brazil. Mem Inst Oswaldo Cruz 1999; 94:121-122.

10. Lee HL, Tadano T. Monitoring resistance gene frequency in Malaysian $C x$ quinquefasciatus Say adults using rapid enzyme microassays. Southeast Asian J Trop Med Public Health 1994; 25:371-373.

11. Gerberg EJ. Manual for mosquito rearing and experimental techniques. Am Mosq Control Assoc 1979; 5:1-124.

12. World Health Organization. Instruciones for determining the susceptibility or resistence of mosquito larvae to insecticides. Vector Biology and Control $1981 ; 81.807: 1-6$

13. Morgan BJT. Analysis of quantal response data. London: Chapman \& Hall; 1992.

14. Dorta DM, Vasuki V, Rajavel A. Evaluation of organophosphorus and synthetic pyrethroid insecticides against six vector mosquitoe species. Rev Saude Publica $1993 ; 27: 391-397$

15. Bisset J, Rodríguez M, Soca A. Cross-resistance to malathion in Cuba Culex quinquefasciatus induced by larval selection with deltamethrin. Med Vet Entomol 1998; 12:109-112.

16. Georghieu G, Pasteur N. Electrophoretic esterase patterns in insecticide-resistat and susceptible mosquitoes. J. Econ Entomol 1978; 71:201-205.

17. Kao LR, Motoyama N, Dauterman WC. Study on hydrolases in various housefly strains and their role in Malathion resistance. Pestic Biochem Physiol 1984; 22:86-92.

18. ScottJG. Cytochrome P450 monooxygenase mediated resistance to insecticides. J Pestic Sci 1996; 21:241-245

19. Vulule JM, Beach FK, Atieli FK, Mcallister JC, Brogdon WG, Roberts JM, et al. Elevated oxidase and esterase levels associated with permethrin tolerance in Anopheles gambiae from Kenyan villages using permethrin-impregnated nets. Med Vet Entomol 1999; 13: 239-244.
20. Yu SJ. Insect glutathione transferases. Zool Stud 1995; 35:9-19.

21. Bracco JE, Barata JMS, Marinotti O. Evaluation of insecticide resistance and Biochemical Mechanisms in a population of Culex quinquefasciatus (Diptera: Culicidae) from São Paulo, Brazil. Mem Inst Oswaldo Cruz 1999; 94:115-120.

22. Campbell WC, Fisher MH, Stapley EO, Albers-Shönberg G, Jacob TA. Ivermectin: a potent antiparasitic agent. Science 1983; 221:823-828.

23. Dybas RA. Abamectin use in crop protection. In: Campbell WC, editor. Ivermectin and abamectin. New York: Springer-Verlag; 1989. p. 287-310.

24. Wislocki PG, Grosso LS, Dybas RA. Environmental aspects to abamectin use in crop protection. In: Campbell WC, editor. Ivermectin and abamectin. New York: Springer-Verlag; 1989. p. 182-200.

25. Freitas RMC, Faria MA, Alves SN, Melo AL. Effects of ivermectin on Culex quinquefasciatus larvae. Rev Inst Med Trop São Paulo 1996; 38:293-297.

26. Alves SN, Serrão JE, Mocelin G, Melo AL. Ivermectin effects on the life cycle and larval fat body of Culex quinquefasciatus (Say, 1823) (Diptera, Culicidae) Braz Arch Biol Technol 2004; 47:433-439.

27. Alves SN, Serrão JE, Melo AL. Alterations in the fat body and midgut of Culex quinquefasciatus larvae following exposure to different insecticides. Micron 2010; 41:592-597.

28. Neves DP, Linardi PM, Cunha HC. Empregro do K-othrine (Decamethrin NRDC-161) no controle de Aedes fluviatilis e de Culex pipiens fatigans: testes de laboratório e campo. Rev Bras Biol 1981; 41:607-614.

29. Dover MJ, Croft BA. Pesticide resistance and public policy. Bioscience $1986 ; 36: 78-85$.

30. Campos J, Andrade CFS. Susceptibilidade larval de duas populações de Aedes aegypti a inseticidas químicos. Rev Saude Publica 2001; 35:232-236. 